\title{
Effect of Acupuncture for Diarrhea-Predominant Irritable Bowel Syndrome: Study Protocol for a Randomized Clinical Trial
}

\section{Ling-Yu Qi}

Beijing University of Chinese Medicine

Jing-Wen Yang

Beijing University of Chinese Medicine

\section{Shi-Yan Yan}

Beijing University of Chinese Medicine

\section{Yan-Fen She}

Hebei University of Chinese Medicine

\section{Hui Hu}

Dongfang Hospital

\section{Ying Li}

Chengdu University of TCM: Chengdu University of Traditional Chinese Medicine

\section{Li-Li Chi}

Shandong University of Traditional Chinese Medicine Affiliated Hospital

\section{Bang-Qi Wu}

First Teaching Hospital of Tianjin University of Traditional Chinese Medicine

Jian-Feng Tu

Beijing University of Chinese Medicine

\section{Li-Qiong Wang}

Beijing University of Chinese Medicine

\section{Cun-Zhi Liu (D lcz_tg@126.com)}

School of Acupuncture-Moxibustion and Tuina, Beijing University of Chinese Medicine https://orcid.org/0000-0001-8031-5667

\section{Research Article}

Keywords: Acupuncture, Diarrhea-predominant Irritable bowel syndrome, Randomized controlled trials, Protocol

Posted Date: January 21st, 2022

DOI: https://doi.org/10.21203/rs.3.rs-710661/v1 
License: (c) (i) This work is licensed under a Creative Commons Attribution 4.0 International License. Read Full License 


\section{Abstract \\ Background}

Diarrhea-predominant irritable bowel syndrome (IBS-D) is the most common subtype of IBS. Acupuncture is commonly used to treat IBS-D, but its effect is uncertain because of the poor quality of prior studies. Therefore, we designed this trial to evaluate the effect and safety of acupuncture for the treatment of IBSD through comparisons with sham acupuncture.

\section{Methods/design:}

This is a large-scale, multi-center, randomized, two-arm interventional clinical trial. Participant will take part in a total of 20 weeks, which contained 3 phases that 2-weeks screening, 6-weeks treatment, 12-week follow-up. Based on the composite response rate of primary endpoint in our pilot study (a sham acupuncture response rate of $27 \%$ and a true acupuncture of approximately $45 \%$ ), 280 randomly allocated participants were planned. Eligible participants will be randomly assigned to true acupuncture group and sham acupuncture group according to the ratio of 1:1, and a total of 15 sessions of treatment overall 6-week treatment period will be brought. The primary endpoint is a composite response rate at week 6 , and the responder is defined as who responses in both abdominal pain intensity and stool consistency. Furthermore, composite response rates at other weeks, IBS Symptom Severity Scale, IBS Quality of Life, Adequate Relief scale and individual IBS symptoms (abdominal pain, bloating, stool frequency) are chosen as secondary endpoints.

\section{Discussion}

This trial may provide high-quality evidence for the efficacy and safety of acupuncture in the treatment of IBS-D. The results of this study will be published in peer-reviewed journals.

\section{Trial registration:}

ClinicalTrials.gov, ChiCTR2100044762. Registered on 26 March 2021.

\section{Background}

Irritable bowel syndrome (IBS) is a globally prevalent disorder characterized by persistent or intermittent abdominal pain, bloating, changes of stool form and frequency and defecation habits. Although IBS is non-fatal, the symptoms impair quality of life and social functioning and lead to a health-care burden. The annual direct and indirect costs associated with IBS are estimated to be up to billions or more in several countries [1-3]. In the absence of distinct biomarkers, IBS is typically diagnosed on the basis of symptom criteria, such as the Rome IV or Rome III criteria. Diarrhea-predominant IBS (IBS-D) is the most 
common subtype when the Rome IV criteria were applied [4] according to a systematic review and metaanalysis of global prevalence of IBS in 2020. Routine clinical treatments for it trend to improve abdominal pain and bowel habit, but often are targeted towards the most troublesome symptom. However, antidiarrhoeals and antispasmodics are used as first-line therapies for predominant stool pattern improvement in IBS, but most randomized controlled trials of these drugs are outdated and are hampered by suboptimal methodology and heterogeneous patient selection. And the occurrence of side effects and adverse events greatly reduced the therapeutic effect and patients' satisfaction, which resulted in a higher demand for alternative therapies [5].

Acupuncture is considered a beneficial alternative treatment for functional gastrointestinal disorders [6]. Although whether acupuncture is an alternative treatment for IBS still remains controversial [7-10], previous studies have shown that acupuncture may affect IBS from the perspectives of the brain-gut axis, gastrointestinal motility, and visceral hypersensitivity [11]. Furthermore, the recent clinical randomized trial supported that acupuncture may be more effective than pinaverium bromide for the treatment of IBSD, with effects lasting up to 12 weeks [12]. Regrettably, efficacy endpoints of most clinical trials of acupuncture for IBS often do not meet current recommendations from the US Food and Drug Administration (FDA) or the European Medicines Agency. We have conducted a pilot clinical randomized trial with a minimum sample size $(n=90)$ in 2020, in which efficacy endpoints meet current recommendations from the FDA were used [13]. For primary endpoint, the composite response rate at week 4 between true acupuncture (specific acupoints and non-specific acupoints) and sham acupuncture (non-acupoints) were $46.67 \%$ and $26.67 \%$, and the line charts trends of all endpoints of specific acupoints acupuncture are consistent and better than sham acupuncture. However, the effective response rates of different acupuncture groups and sham acupuncture group have no statistical significance, and we suggest that the insufficient sample size cannot be ignored. Therefore, a large-sample, multicenter randomized controlled trial with high methodologic quality was considered necessary.

Accordingly, we designed the large-scale trial in order to provide substantive evidence on acupuncture as a reliable option, and evaluate the effect and safety of acupuncture for the treatment of IBS. Our hypothesis was that 6-week acupuncture treatment would be more effective than sham acupuncture in the alleviation of IBS symptoms.

\section{Methods/design}

\section{Trial design}

This is a large-scale, multi-center, randomized, two-arm interventional clinical trial (Figure 1). Participant will take part in a total of 20 weeks, which contained 3 phases that 2-weeks screening (week -2, -1), 6weeks treatment (week 1 - 6), 12-week follow-up (week 7 - 18). Participants will be recruited in 5 Grade III Level A hospitals in China: (I) The Affiliated Hospital of Hebei University of Chinese Medicine; (II) the First Teaching Hospital of Tianjin University of Traditional Chinese Medicine; (III) the Teaching Hospital of Chengdu University of Traditional Chinese Medicine; (IV) the Affiliated Hospital of Shandong University of 
Traditional Chinese Medicine; and (V) the Dongfang Hospital Beijing University of Chinese Medicine. The study protocol was approved by the Medical Ethics Committee of Beijing University of Chinese before the study started. All personnel participating in this trial will be uniformly trained on the content of trial.

\section{Participants}

Participants will be recruited by the clinical recruiters using advertisements (eg, flyers in physician offices, articles on WeChat official accounts). Data of trial will be collected by the REDCap electronic data capture (EDC) system [14]. Every participant will sign an informed consent form before randomization.

\section{Inclusion criteria}

(囚) Aged between 18 and 75 years (either sex);

(『) Fulfilled Rome IV diagnostic criteria for IBS-D;

$(\Downarrow)$ Type 6 or 7 of the Bristol Stool Form Scale appeared for at least 4 days and type 1 or 2 appeared for less than 4 days in last 2 weeks; The average score of daily abdominal pain was $\geq 3$ in the last week;

$(\Downarrow)$ No treatment of acupuncture in the last 6 months;

(『) No use of antidepressant or IBS medication within 2 weeks before treatment, including traditional Chinese medicine (TCM) or proprietary Chinese medicine, antidiarrheal, antispasmodic, intestinal antibiotics, probiotics and so on.

\section{Exclusion criteria}

(『) Participants with inflammatory bowel disease, microscopic colitis, celiac disease, Crohn's disease and other organic bowel diseases (age $\geq 50$ years or have the following alarm signs will be required to provide colonoscopy report within nearly 2 years: unexplained weight loss (weight loss $>10 \%$ within 3 months); hematochezia caused by non-hemorrhoids or anal fissure; nocturnal diarrhea; family history of colorectal cancer);

(邓) Diabetes mellitus and abnormal thyroid function;

(『) Severe acute or chronic organic diseases, kidney or liver diseases;

( $₫)$ History of previous abdominal surgery (appendectomy, hemorrhoidectomy, or polypectomy greater than 3 months post-surgery are allowed.);

(邓) Pregnancy or lactation;

( $\mathbb{Q})$ History of alcohol and drug abuse;

(『) Participate in other clinical trials. 


\section{Interventions}

True acupuncture (TA) group and sham acupuncture (SA) group will be set in this trial. The acupuncturists will be required to have a minimum licensed time of 3 years. Participants will receive 15 sessions of treatment overall 6-week treatment period. For first 3 weeks of 6-week treatment period, each treatment will be performed 3 times a week (once every other day); for last 3 weeks of 6-week treatment period, each treatment will be performed 2 times a week (once every three days). Single-use sterile needles $(0.30 \mathrm{~mm}$ in diameter and $40 \mathrm{~mm}$ in length or $0.30 \mathrm{~mm}$ in diameter and $25 \mathrm{~mm}$ in length; Hwato, Suzhou, China) will be used in TA group. Blunt-tipped placebo needles will be used (similar to the Streitberger design) which can provide participant-blinding effects with a similar appearance to conventional needles but no skin penetration [15-16]. Adhesive pads will be placed on puncture points in both groups. The use of blunt-tipped placebo needles and adhesive pads is to help maximize blinding of participants in SA group, and adhesive pads also has the function of fixing blunt-tipped placebo needles.

\section{TA group}

According to different types of TCM syndrome, five fixed acupoints and one of three optional acupoint will be chose for the treatment of participants in TA group (Table 1). After sterilization, the acupuncturist will insert single-use sterile needles into the deep tissue layers through adhesive pads of acupoints. Following needle insertion, small, equal manipulations of twirling, lifting, and thrusting will be performed on all needles to reach de qi (a component sensation, including soreness, numbness, distension and heaviness). The retention time of single-use sterile needles will be 30 minutes, during which the operation of de qi will also be performed every 10 minutes during the needle retention period.

Table 1 Locations of acupoints for TA group 


\begin{tabular}{|c|c|c|}
\hline & Acupoint & Location \\
\hline \multirow[t]{5}{*}{$\begin{array}{l}\text { Fixed acupoints } \\
\text { of SA group }\end{array}$} & $\begin{array}{l}\text { Tianshu } \\
\text { (ST25) }\end{array}$ & On the horizontal line of the navel, 2 cun $^{\mathrm{a}}$ beside the anterior midline \\
\hline & $\begin{array}{l}\text { Zhongwan } \\
\text { (RN12) }\end{array}$ & $\begin{array}{l}\text { On the anterior midline of upper abdomen, } 4 \text { cun superior to the } \\
\text { navel }\end{array}$ \\
\hline & $\begin{array}{l}\text { Guanyuan } \\
\text { (CV4) }\end{array}$ & On the anterior midline of abdomen, 3 cun inferior to the navel \\
\hline & $\begin{array}{l}\text { Zusanli } \\
\text { (ST36) }\end{array}$ & $\begin{array}{l}3 \text { cun directly below ST } 35 \text {, and one finger-breadth lateral to the } \\
\text { anterior border of the tibia }\end{array}$ \\
\hline & $\begin{array}{l}\text { Shangjuxu } \\
\text { (ST37) }\end{array}$ & $\begin{array}{l}\text { On the anterolateral aspect of the leg, } 6 \text { cun inferior to the ST35, and } \\
\text { one finger-breadth lateral to the anterior border of the tibia }\end{array}$ \\
\hline \multirow{3}{*}{$\begin{array}{l}\text { Optional } \\
\text { acupoints of } \\
\text { SA group }\end{array}$} & $\begin{array}{l}\text { Taichong } \\
\text { (LR3) }\end{array}$ & $\begin{array}{l}\text { In the depression anterior to the junction of the first and second } \\
\text { metatarsal bones }\end{array}$ \\
\hline & $\begin{array}{l}\text { Sanyinjiao } \\
\text { (SP6) }\end{array}$ & $\begin{array}{l}\text { On the tibial aspect of the leg, posterior to the medial border of the } \\
\text { tibia, } 3 \text { cun superior to the prominence of the medial malleolus }\end{array}$ \\
\hline & $\begin{array}{l}\text { Neiting } \\
\text { (ST44) }\end{array}$ & $\begin{array}{l}\text { On the instep, between the second and third toes of the red and } \\
\text { white flesh behind the webbed margin }\end{array}$ \\
\hline
\end{tabular}

${ }^{\mathrm{a}} 1$ cun $(\approx 20 \mathrm{~mm})$ is defined as the width of the interphalangeal joint of the participant's thumb

\section{SA group}

Five non-acupoints that away from meridians or conventional acupoints will be selected for the treatment of SA group (Table 2). Unlike the TA group, the SA group will not perform manual operations for needles after needles insertion and pretend to perform the operation of de qi every 10 minutes during needle retention time.

Table 2 Locations of acupoints for SA group 


\begin{tabular}{|c|c|}
\hline $\begin{array}{l}\text { Non- } \\
\text { acupoint }\end{array}$ & Location \\
\hline $\begin{array}{l}\text { Non- } \\
\text { acupoint } \\
1\end{array}$ & $\begin{array}{l}\text { On the abdomen, } 2 \text { cun }^{\mathrm{a}} \text { superior to anterior superior iliac spine, between the gallbladder } \\
\text { meridian and the spleen meridian }\end{array}$ \\
\hline $\begin{array}{l}\text { Non- } \\
\text { acupoint } \\
2\end{array}$ & $\begin{array}{l}\text { On the abdomen, } 2 \text { cun inferior to navel, } 1 \text { cun beside the anterior midline, between the } \\
\text { kidney meridian and the stomach meridian }\end{array}$ \\
\hline $\begin{array}{l}\text { Non- } \\
\text { acupoint } \\
3\end{array}$ & $\begin{array}{l}\text { On the lateral aspect of the leg, } 3 \text { cun inferior to GB34, between gallbladder meridian and } \\
\text { bladder meridian }\end{array}$ \\
\hline $\begin{array}{l}\text { Non- } \\
\text { acupoint } \\
4\end{array}$ & $\begin{array}{l}\text { On the leg, } 2 \text { cun superior to the medial malleolus, in the middle of the medial tibia, } \\
\text { between the liver meridian and the spleen meridian }\end{array}$ \\
\hline $\begin{array}{l}\text { Non- } \\
\text { acupoint } \\
5\end{array}$ & $\begin{array}{l}\text { On the leg, the midpoint of the line between GB } 40 \text { and ST41, between the gallbladder } \\
\text { meridian and the stomach meridian }\end{array}$ \\
\hline
\end{tabular}

${ }^{a} 1$ cun $(\approx 20 \mathrm{~mm})$ is defined as the width of the interphalangeal joint of the participant's thumb

All participants will be advised to keep their routine lifestyles and diets during the study procedure. Loperamide (Imodium, Xian Janssen Pharmaceutical Ltd., China), will be used as rescue medication under the guidance of gastroenterologists whenever necessary. The medication status and other nonirritable bowel syndrome drugs applications of participants will be strictly recorded during the trial.

\section{Sample size}

The sample size calculation was based on the composite response rate of primary endpoint in our pilot study (not yet published). The results of our pilot study showed that a sham acupuncture response rate of $27 \%$ and a true acupuncture of approximately $45 \%$ were estimated for the primary endpoint. A sample size of 222 evaluable participants was calculated to be needed for $80 \%$ power. With an estimated dropout rate of $20 \%$ and the balance of recruitment quotas in 5 sites, 280 randomly allocated participants were planned.

\section{Randomization and Blinding}

Eligible participants will be randomly assigned to TA group and SA group according to the ratio of 1:1 by central stratified block randomization in REDCap EDC system with a dynamic block size. The randomization sequence was created by a biostatistician who did not participate in this trial. The REDCap EDC system permissions vary depending on the role of the personnel. Only the acupuncturist and his or her assistant can obtain the randomization grouping information. The clinical recruiters, outcome assessors, data managers, and statisticians will be blinded to group allocation.

\section{Outcomes and Follow-Up}


The primary endpoint is a composite response rate at week 6 in accordance with FDA recommendations. A participant is defined as a composite responder if the participant is a responder in both abdominal pain intensity and stool consistency. Abdominal pain intensity responder defined as: decrease in weekly average of worst abdominal pain in past 24 hours score of at least $30 \%$ compared with baseline; Stool consistency responder defined as: decrease at least $50 \%$ in the number of days per week with at least one stool that has a consistency of Type 6 or 7 compared with baseline.

The secondary endpoints also include composite response rates at other weeks (weeks 2, 4, 10, 14, and 18). IBS Symptom Severity Scale (IBS-SSS) is used to evaluate the overall symptoms of IBS, which comprises 5 domains (severity of abdominal pain, frequency of abdominal pain, severity of abdominal distension, degree of dissatisfaction with defecation habits and interference with the quality of life) that are scored from 0 to 100, and total score range 0 to 500). A decrease of 50 points is considered a value with clinical improvement significance [17]. IBS Quality of Life (IBS-QoL) comprises 34 IBS-specific items which divides 8 variables: health worries, food avoidance, body image, dysphoria, interference with activity, social reactions, sexual activity and relationships [18]. The IBS-QoL score will transformed into a 0-100 scale using the follow formula: (the actual raw score-the lowest possible score)/possible score rangex 100 . The Patient Health Questionnaire-9 (PHQ-9) is a diagnostic instrument for common mental disorders, of which scores range from 0 to 27 and the mental state is inversely proportional to the total score of it [19]. Individual IBS symptoms (abdominal pain, abdominal bloating, stool frequency) will also be collected and analyzed. Adequate Relief (AR) scale will be used to confirm whether participants have an adequate relief by a two-category (yes or no) answer. IBS-SSS, IBS-QoL, PHQ-9, AR scale and individual symptoms will be assessment at weeks $2,4,6,10,14$, and 18 .

For blinding assessment, all participants will be asked to guess which treatment they received at the end of the last treatment in the week 6. Additionally, Credibility/ Expectancy Questionnaire within 5 minutes will be used to evaluate credibility and expectancy of participants after the first treatment [20]. The schedule of enrolment, intervention, and assessments is shown in Figure 2. The incidence of AEs and any use of loperamide will be recorded at 6-week treatment and 12-week follow-up.

\section{Human body data retention}

Considering that the different types of testing instruments in different hospitals will affect the results of sample analysis, we will carry out sample retention work from Dongfang Hospital Beijing University of Traditional Chinese Medicine. Participants will be asked whether willing to offered stool sample and functional Magnetic Resonance Imaging (fMRI) data at baseline and the end of intervention. Sample retention aims to observe the relevant changes before and after intervention. The collected stool samples will be used for the detection of gut microbial diversity and richness, and brain imaging data will be used to compare the differences in brain activity areas.

\section{Data management and Monitoring}


All researchers will be required to strictly protect the individual privacy of the participants. The electronic Case Report Form (eCRF) will be used to input general information and evaluation content related to the trial. Dynamic management will be performed to ensure complete, timely and accurate data collection using the verification function in eCRF. And the researchers will no longer be able to modify content of eCRF, because the database will be locked by the data management team when the trial is completed. In order to facilitate the elderly or other participants who are inconvenient to use electronic devices, we will also provide corresponding paper materials (e.g., defecation diary cards). Both paper files and electronic documents will be preserved for at least 5 years after publication. If readers and reviewers have any questions, they can contact the corresponding author for access to the original data.

Experts in acupuncture, gastroenterology, methods, and statistics will review and revise the protocol. Before the trial, all researchers will be trained on standard operating procedures (such as screening patients, acupuncture, filling in eCRF, evaluation results, and data management). Online monitoring and on-site monitoring will be adopted in this trial. All modifications of the data can be traced through the eCRF. Appropriate communication will be maintained with the patients to strengthen their compliance and ensure data integrity. In addition, a Data Safety and Monitoring Board (DSMB) will be independently established to review and interpret the data of trial. The Board will review the progress of the trial, independently of the investigators, and decide whether the trial needs to be terminated early solely on the basis of adverse events.

\section{Statistical Analyses}

All analyses related to treatment efficacy will based on the intention-to-treat (ITT) population, defined as all randomly assigned participants with baseline data. Safety analyses will be based on the safety population who have received at least one treatment after randomization and have safety indicator records. Missing data will be imputed using multiple imputation.

Continuous data will be represented by mean \pm standard deviation $(\mathrm{M} \pm \mathrm{SD})$ or median combined with interquartile range, whereas categorical data will be represented by frequency, constituent ratio and percentage. For primary endpoint, the Chi-square test will be used for the comparison of two groups. For secondary endpoints, Student's t test or Wilcoxon rank-sum test will used to assess continuous variables between groups comparisons, and Chi-square test or Fisher exact tests will be used to evaluate categorical variables between-groups. The safety population will be used to analyze the incidence of AEs between groups. The $P$ value less than 0.05 will be considered to be statistically significant. All analyses will be performed using SAS 9.3.

\section{Adverse Event}

Adverse events (AEs) will be appropriately assessed, managed, and recorded by the acupuncturists and related clinical specialists. We have set a specific questionnaire for recording AEs in real time by REDCap EDC system. All AEs will be dealt with symptomatically. If the acupuncturist is unable to handle it, it is 
necessary to consult specialists of the corresponding discipline for consultation and treatment. Common acupuncture-related AEs include subcutaneous hematoma, continuous post-needling pain, dizziness, etc.

\section{Discussion}

Although IBS has been extensively studied, its uncertain pathophysiology and unsatisfactory treatments account a growing demand for alternative treatments, including acupuncture. This large-scale trial will evaluate the effect and safety of acupuncture for the treatment of IBS.

For this trial, the application of blunt-tipped placebo needles, adhesive pads, and REDCap EDC system will enable the trial progress to better meet the methodological demand for randomization, allocation concealment, and blinding of participants, clinical recruiters, outcome assessors, data managers, and statisticians. Meanwhile, we will assess participant expectation and blinding which seems have influence on acupuncture effects. Additionally, the primary endpoint compliances with FDA standards which will make the trial results more convincing. To the best of our knowledge, no prior RCT using FDArecommended indicators to compare the effect between true acupuncture and sham acupuncture on IBSD.

Intestinal microbiota is currently a hot area of research. Studies on intestinal microbiota of IBS found that patients with IBS had higher abundance of Phylum Firmicutes and lower abundance of Bacteroides compared with healthy controls [21-22], and the fecal microecological diversity of various subtypes of IBS patients was different [23]. In addition, Quigley et al. have suggested that the over reproduction of intestinal microbiota may be an important factor in the induction of IBS [24]. Changes in the intestinal microbiota are closely related to IBS, however, there are no studies to confirm the regulatory effect of acupuncture on intestinal flora of IBS. Moreover, symptoms of IBS are considered to be related to disorders of the nervous system of the brain, and fMRI is useful to explore abnormal brain functions. Some researchers have used fMRI to investigate the potential mechanism of IBS, but the results are not completely consistent [25-26]. Therefore, we will retain their stool samples and fMRI data according to the wishes of the participants to explore the possible mechanisms of acupuncture treatment of IBS.

There are several limitations in this trial. First, previous study put forward that 6 sessions treatment of acupuncture over 3 weeks may have been insufficient, [27], so we chose the 15 sessions of treatment overall 6-week treatment period to achieve maximum effect from acupuncture. This may be more complex with actual clinical practice in China, but it may be difficult to implement in other countries due to treatment costs, commuting and travel time. Therefore, we have made modifications for per week sessions (i.e., 3 or 2 sessions of treatment per week) to explore a standardized acupuncture protocol for IBS. Second, this trial is failed to blind acupuncturists which may affect the effect of interventions between groups. Third, although interventions in SA group have no operation of skin penetration, it cannot eliminate the placebo effect completely, which should be taken into consideration when drawing conclusions.

\section{Trial status}

Page $11 / 18$ 
This trial is currently recruiting patients.

\section{Registration}

ClinicalTrials.gov, NCT03274713. Registered on 26 March 2021.

\section{Protocol}

Version 1.0, 26 March 2021.

\section{Abbreviations}

\section{REDCap}

Research electronic data capture; IBS-D:diarrhea-predominant irritable bowel syndrome; IBS:irritable bowel syndrome; FDA:Food and Drug Administration; EDC:electronic data capture; TCM:traditional Chinese medicine; TA:true acupuncture; SA:sham acupuncture; IBS-SSS:IBS Symptom Severity Scale; IBSQoL:IBS Quality of Life; PHQ-9:Patient Health Questionnaire-9; AR:Adequate Relief; fMRI:functional Magnetic Resonance Imaging; eCRF:electronic Case Report Form; DSMB:Data Safety and Monitoring Board; ITT:the intention-to-treat; $\mathrm{M} \pm \mathrm{SD}$ :mean \pm standard deviation; AEs:Adverse events.

\section{Declarations}

\section{Ethics approval and consent to participate}

The study was performed in accordance with the Declaration of Helsinki and was approved by the Medical Ethics Committee of Beijing University of Chinese. Written informed consent will be obtained from all participants.

\section{Consent for publication}

Not applicable.

\section{Availability of data and material}

The corresponding author can be contacted to obtain data according to reasonable requirements.

\section{Competing interests}

The authors have no conflicts of interest to declare.

\section{Funding}

This study was supported by the National Key Research and Development Program of China (No. 2019YFC1712103). The sponsor has no role in designing the trial, collection, management, analysis, and interpretation of data and the decision to write a report or submit a report for publication. 


\section{Authors' contributions}

Conception and design: LY Qi, CZ Liu; Administrative support: JW Yang, CZ Liu; Provision of study materials or patients: YF She, H Hu, Y Li, LL Chi, BQ Wu, LQ Wang; Collection and assembly of data: LY Qi, SY Yan; Data analysis and interpretation: LY Qi, SY Yan, JF Tu; Manuscript writing: LY Qi, CZ Liu; All authors read and approved the final manuscript.

\section{Acknowledgements}

The authors are grateful to all medical workers who assisted on this trial.

\section{Author details}

${ }^{1}$ International Acupuncture and Moxibustion Innovation Institute, Beijing University of Chinese Medicine, Beijing 100029, China; Acupuncture Research Center, School of Acupuncture-Moxibustion and Tuina, Beijing University of Chinese Medicine, Beijing 100029, China. Email: qly66love@163.com;

2 International Acupuncture and Moxibustion Innovation Institute, Beijing University of Chinese Medicine, Beijing 100029, China; Acupuncture Research Center, School of Acupuncture-Moxibustion and Tuina, Beijing University of Chinese Medicine, Beijing 100029, China. Email: yangjw0626@126.com;

${ }^{3}$ International Acupuncture and Moxibustion Innovation Institute, Beijing University of Chinese Medicine, Beijing 100029, China; Acupuncture Research Center, School of Acupuncture-Moxibustion and Tuina, Beijing University of Chinese Medicine, Beijing 100029, China. Email: yanshiyan0927@sina.com;

${ }^{4}$ School of Acupuncture-Moxibustion and Tuina, Hebei University of Chinese Medicine, Shijiazhuang 050299, China; Email: sheyanfen@163.com;

${ }^{5}$ Department of Acupuncture and Moxibustion, Dongfang Hospital, Beijing University of Chinese Medicine, Beijing 100029, China; Email: dfzjhh@163.com—

${ }^{6}$ School of Graduate, Chengdu University of Chinese Medicine, Chengdu 610075, China; Email: liying@cdutcm.edu.cn;

${ }^{7}$ Department of Spleen and Stomach, the Affiliated Hospital of Shandong University of Traditional Chinese Medicine, Jinan 250011, China; Email: chililiyl@163.com;

${ }^{8}$ National Acupuncture and moxibustion Clinical Medical Research Center, the First Teaching Hospital of Tianjin University of Traditional Chinese Medicine, Tianjin 300193, China. Email: wbqwbq1980@outlook.com;

${ }^{9}$ International Acupuncture and Moxibustion Innovation Institute, Beijing University of Chinese Medicine, Beijing 100029, China; Acupuncture Research Center, School of Acupuncture-Moxibustion and Tuina, 
Beijing University of Chinese Medicine, Beijing 100029, China. Email: tujianfeng1@126.com;

10 International Acupuncture and Moxibustion Innovation Institute, Beijing University of Chinese Medicine, Beijing 100029, China; Acupuncture Research Center, School of Acupuncture-Moxibustion and Tuina, Beijing University of Chinese Medicine, Beijing 100029, China. Email: wangliqiongwork@163.com;

11 International Acupuncture and Moxibustion Innovation Institute, Beijing University of Chinese Medicine, Beijing 100029, China; Acupuncture Research Center, School of Acupuncture-Moxibustion and Tuina, Beijing University of Chinese Medicine, Beijing 100029, China. Email: Icz_tg@126.com.

*Correspondence to: Cun-zhi Liu. Email: Icz_tg@126.com.

\section{References}

1. Flacco ME, Manzoli L, De Giorgio R, Gasbarrini A, Cicchetti A, Bravi F, et al. Costs of irritable bowel syndrome in European countries with universal healthcare coverage: a meta-analysis. Eur Rev Med Pharmacol Sci. 2019;23:2986-3000.

2. Zhang F, Wei X. Li Chun-Yan, Li Shu-Chuen. Economic burden of irritable bowel syndrome in China. World J Gastroenterol. 2016;22:10450-60.

3. Peery Anne F, Crockett Seth D, Murphy Caitlin C, Lund Jennifer L, Dellon Evan S, Williams J, Lucas, et al. Burden and Cost of Gastrointestinal, Liver, and Pancreatic Diseases in the United States: Update 2018. Gastroenterology. 2019;156:254-72.e11.

4. Oka Priya P, Heather B, Brigida, Black Christopher J, Savarino Edoardo V, Ford Alexander C. Global prevalence of irritable bowel syndrome according to Rome III or IV criteria: a systematic review and meta-analysis. Lancet Gastroenterol Hepatol. 2020;5:908-17.

5. Tillisch K. Complementary and alternative medicine for functional gastrointestinal disorders. Gut. 2006;55:593-6.

6. Li Hui H, Tian Xu, Qian, Li Z, Liu Y, Li Fang, Yang Bo-Feng, et al. Acupuncture and regulation of gastrointestinal function. World J Gastroenterol. 2015;21:8304-13.

7. Chao Guan-Qun. Shuo Z. Effectiveness of acupuncture to treat irritable bowel syndrome: a metaanalysis. World J Gastroenterol. 2014;20:1871-7.

8. Manheimer Eric C, Ke WL, Susan ML, Shih S, Xueyong, Berman Brian M, et al. Acupuncture for treatment of irritable bowel syndrome. Cochrane Database Syst Rev. 2012;undefined(5):CD005111.

9. Manheimer Eric WL, Susan C, Ke LS, Min S, Xueyong, Berman Brian M, et al. Acupuncture for irritable bowel syndrome: systematic review and meta-analysis. Am J Gastroenterol. 2012;107:835 - 47; quiz 848.

10. Lim B, Manheimer E, Lao L, Ziea E, Wisniewski J, Liu J, et al. Acupuncture for treatment of irritable bowel syndrome. Cochrane Database Syst Rev. 2006; undefined:CD005111. 
11. Ma X-P, Jue H, Cai-Ping A, Dan Z, Huang Y, Huan-Gan Wu, et al. Acupuncture-moxibustion in treating irritable bowel syndrome: how does it work. World J Gastroenterol. 2014;20:6044-54.

12. Pei Lixia G, Hao G, Jing Y, Guohui W, Lin S, Rongrong, et al. Effect of Acupuncture in Patients With Irritable Bowel Syndrome: A Randomized Controlled Trial. Mayo Clin Proc. 2020;95:1671-1683.

13. Qi L-Y, Yu W, Li-Qiong W, Guang-Xia SYan-Fen,S, Ying L, et al. Acupuncture for the treatment of diarrheal-predominant irritable bowel syndrome: study protocol for a pilot randomized controlled trial. Trials. 2021;22:253.

14. Harris Paul A, Taylor Robert T, Robert P, Jonathon G, Nathaniel, Conde Jose G. Research electronic data capture (REDCap)--a metadata-driven methodology and workflow process for providing translational research informatics support. J Biomed Inform. 2009;42:377-81.

15. Streitberger K, Kleinhenz J. Introducing a placebo needle into acupuncture research. Lancet. 1998;352:364-5.

16. Liu Baoyan Xu, Huanfang M, Rui M, Qian Y, Shiyan L. Zhishun. Effect of blinding with a new pragmatic placebo needle: a randomized controlled crossover study. Med (Baltim). 2014;93:e200.

17. Francis $\mathrm{CY}$, Morris J, Whorwell PJ. The irritable bowel severity scoring system: a simple method of monitoring irritable bowel syndrome and its progress. Aliment Pharmacol Ther. 1997;11:395-402.

18. Patrick DL, Drossman DA, Frederick IO, DiCesare J, Puder KL. Quality of life in persons with irritable bowel syndrome: development and validation of a new measure. Dig Dis Sci. 1998;43:400-11.

19. Jeffery IB, O'Toole PW, Öhman L, Claesson Marcus J, Deane Jennifer, Quigley Eamonn MM, et al. An irritable bowel syndrome subtype defined by species-specific alterations in faecal microbiota. Gut. 2012;61(7):997-1006.

20. Kroenke K, Spitzer RL, Williams JB. The PHQ-9: validity of a brief depression severity measure. J Gen Intern Med. 2001;16:606-13.

21. Devilly GJ, Borkovec TD. Psychometric properties of the credibility/expectancy questionnaire. J Behav Ther Exp Psychiatry. 2000;31:73-86.

22. Rajilić-Stojanović M, Biagi E, Heilig HG, Kajander Kajsa, Kekkonen Riina A, Tims S, et al. Global and deep molecular analysis of microbiota signatures in fecal samples from patients with irritable bowel syndrome. Gastroenterology. 2011;141(5):1792-801.

23. Carroll I, Ringel-Kulka T, Keku T, Chang Young-Hyo P, Christopher D, Sartor R, Balfour, et al. Molecular analysis of the luminal- and mucosal-associated intestinal microbiota in diarrhea-predominant irritable bowel syndrome. Am J Physiol Gastrointest Liver Physiol. 2011;301(5):G799-807.

24. Quigley EM, Craig OF. Irritable bowel syndrome. update on pathophysiology and management. Turk J Gastroenterol. 2012;23(4):313-22.

25. Ma Kai L, Yongkang S, Wei S, Jianhua L, Jing F, Xiaokun, et al. Brain Functional Interaction of Acupuncture Effects in Diarrhea-Dominant Irritable Bowel Syndrome. Front Neurosci. 2020;14:608688. 
26. Zhu Yi Wu, Zhiyuan M, Xiaopeng L, Huirong B, Chunhui Y, Ling, et al. Brain regions involved in moxibustion-induced analgesia in irritable bowel syndrome with diarrhea: a functional magnetic resonance imaging study. BMC Complement Altern Med. 2014;14:500.

27. Lembo AJ, Conboy L, Kelley JM, Schnyer Rosa S, McManus Claire A, Quilty Mary T, et al. A treatment trial of acupuncture in IBS patients. Am J Gastroenterol. 2009;104(6):1489-97.

\section{Figures}




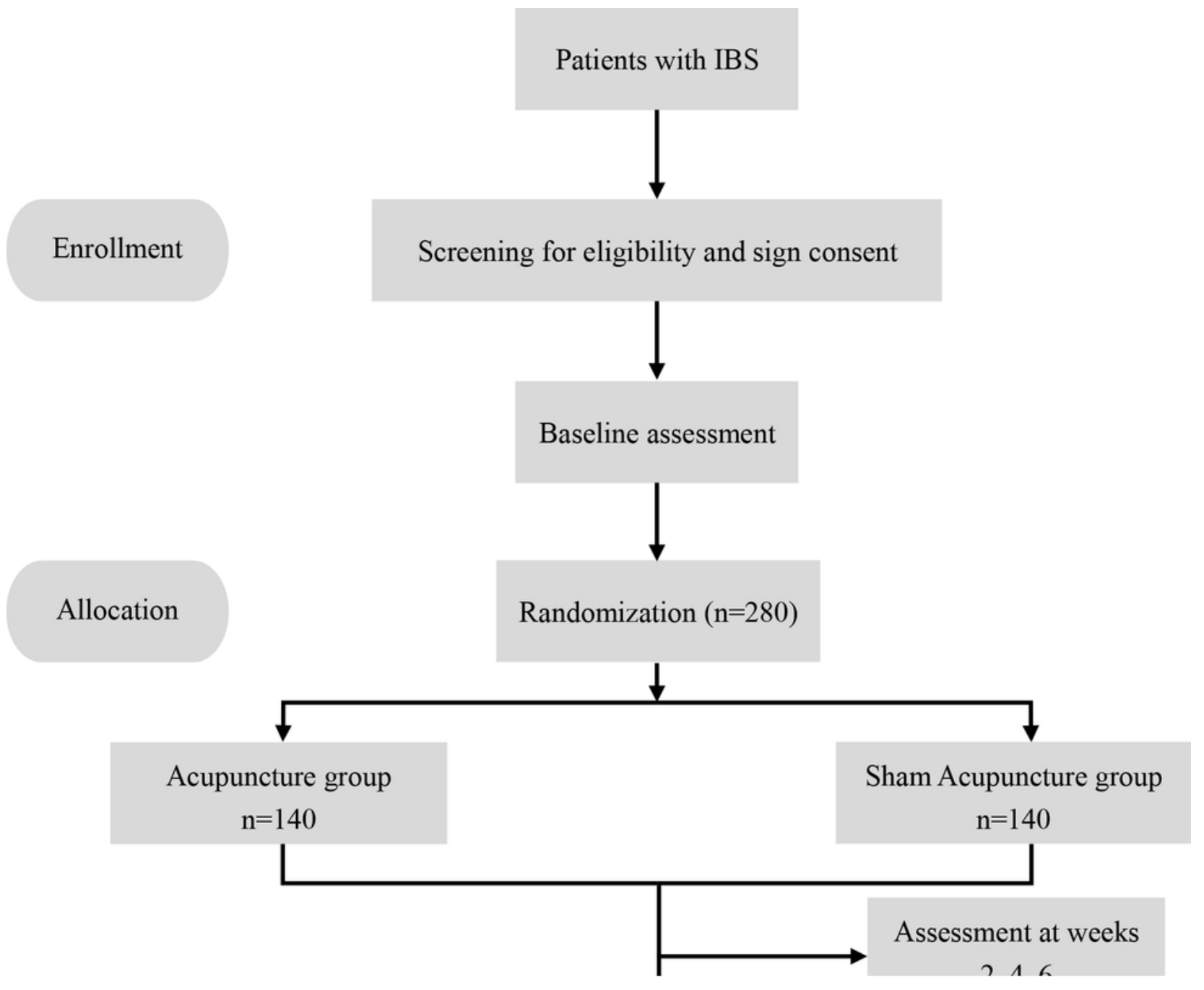

\section{Figure 1}

The flow diagram of this trial.

Figure 2 
Schedule of enrollment, intervention and assessments.

\section{Supplementary Files}

This is a list of supplementary files associated with this preprint. Click to download.

- SPIRITChecklist.docx 